\title{
Libreflix: A Peer-to-Peer On-demand Video Platform for Free Streaming
}

\author{
Guilmour Rossi \\ Universidade Tecnológica Federal do Paraná (UTFPR) \\ Curitiba, Brasil \\ guilmour@alunos.utfpr.edu.br
}

\author{
Luiz Gomes-Jr \\ Universidade Tecnológica Federal do Paraná (UTFPR) \\ Curitiba, Brasil \\ gomesjr@dainf.ct.utfpr.edu.br
}

\begin{abstract}
On-demand video streaming platforms are becoming one of the most important means to publish and consume multimedia content on the internet. The platforms are usually owned by corporations that charge for content access and implement restrictive copyright policies. While the platforms are beneficial, in many aspects, to the multimedia industry and consumers, they can be too restrictive for independent content creators and users with limited resources. This paper presents Libreflix, an on-demand video platform for free publication and consumption of content. Libreflix is intended for aggregation of content with permissive licences, fostering collaboration, community engagement and democratization of knowledge in the internet. This paper describes Libreflix's architecture, the main functionalities implemented in the platform, and discusses important aspects and lessons learned during its ongoing development.
\end{abstract}

\section{KEYWORDS}

streaming platform, free streaming, digital culture, peer-to-peer distribution

\section{INTRODUCTION}

On-demand video platforms have become one of the most popular means to distribute and access multimedia content. A Cisco report [2] estimates that in 2022 video streaming will account for $82 \%$ of all internet traffic. The content offered in typical platforms is, for the most part, comprised of works with all rights reserved by the copyright holder. This content is managed by media companies such as Netflix and Amazon that charge a fee for users to access their collections.

The copyright and financial barriers can be too restrictive for creators and consumers to participate in this cultural development. Following the footsteps of the Free Software movement and the Creative Commons goals, it is important to guarantee free (as in Freedom) access to multimedia works for both consumers and creators without the infringement of copyright laws. Free access to the digital culture is an important step towards the democratization of knowledge.

The goal of this paper is to present, discuss and validate the $\mathrm{Li}$ breflix platform for on-demand video. Libreflix was created in 2017 to catalog multimedia works that can be freely transmitted over the internet (i.e. without copyright restrictions). The platform aims

In: XVIII Workshop de Ferramentas e Aplicações (WFA 2019), Rio de Janeiro, Brasil Anais Estendidos do Simpósio Brasileiro de Sistemas Multimídia e Web (WebMedia). Porto Alegre: Sociedade Brasileira de Computação, 2019.

ISSN 2596-1683 at fostering multimedia culture in online communities, becoming a reference point for free content consumption and free content publication. The Librefix project has deployed several features to address its goals, such as peer-to-peer (P2P) content transmission (Section 3), several user-centered content discovery functionalities and tools for community engagement (Section 4). The paper also discusses important questions related to financing (of the platform and creators), source-code licensing, lessons learned and perspectives (Section 5).

\section{RELATED WORK}

\subsection{On-demand video}

On-demand video services offer catalogs of multimedia content that can be accessed at any time, providing a flexible and convenient user experience [7]. The capitalization model varies, with monthly subscriptions and pay-per-view being the most common schemes.

A key aspect underlying these services is content delivery, usually handled by data streaming protocols [6]. The protocols allow users to start consuming the content as soon as the first packages arrive, avoiding long downloads and waiting times.

\section{$2.2 \quad$ Free Streaming}

The Free Streaming model [10] advocates that the freedom in multimedia streaming distribution is enabled by tools (software programs, platforms, digital archives, etc.) built following the free software [12] guidelines; furthermore, the digital content itself must be amenable to be freely transmitted on the internet without violating the work's copyright. Access must also be free of charge or any other barriers.

The Creative Commons initiative ${ }^{1}$ offers permissive licensing for multimedia content, such as share with Attribution (CC BY), which allows distribution, remixing, and adaptation of content as long as the original creator is properly credited. When a content intended for free distribution is published in a proprietary platform (such as YouTube) that prevents users from downloading the content, the permissiveness of the license is only partially fulfilled.

In this context, the Free Streaming movement emphasizes that comprehensive freedom cannot be accomplished by permissive licensing alone - the concept of freedom must also be ingrained between the mean of access and the transmitted content.

\subsection{Peer-to-peer distribution}

Currently, most of the streaming on the internet is based on the client-server model, where a client connects to the server to retrieve the media content. The Peer-to-Peer (P2P) model that gained

\footnotetext{
${ }^{1}$ https://creativecommons.org
} 


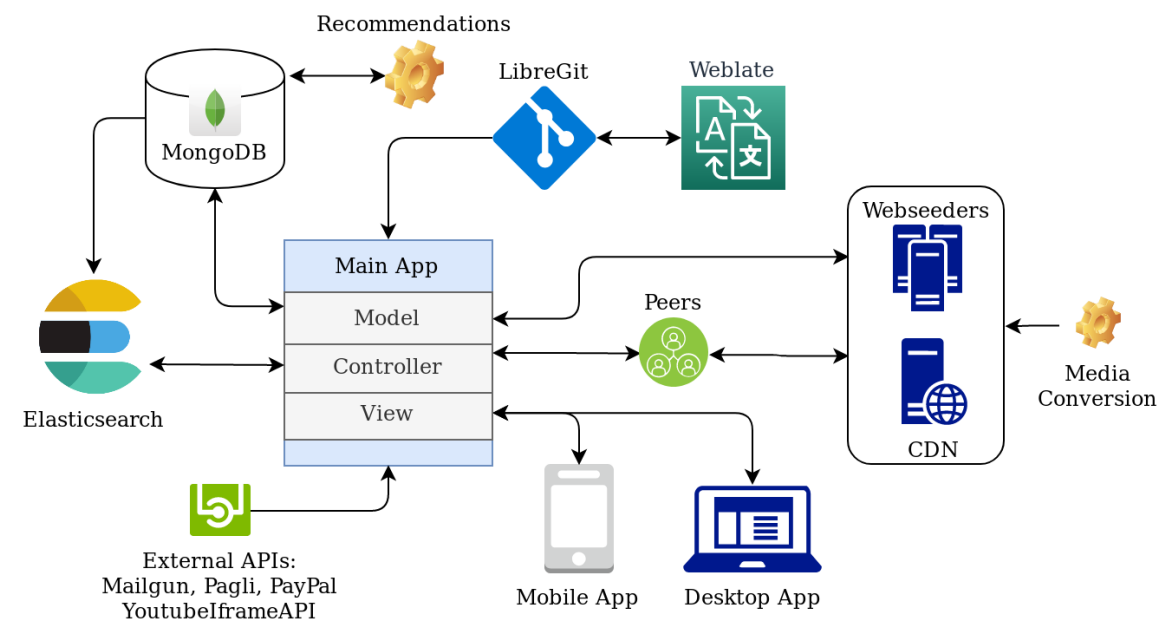

Figure 1: Libreflix architecture

traction in the 2000s, in contrast, considers users (peers) as part of the distribution infrastructure. In a P2P network any peer can serve the content previously downloaded to any other peer. This approach makes the distribution process more reliable and decentralized, reducing the load and importance of dedicated content servers.

WebTorrent [1] is an open source JavaScript library that offers P2P functionalities based on the popular BitTorrent protocol. WebTorrent uses the Web Real-Time Communication (WebRTC) suite of protocols, a W3C standard for client-to-client communication running over web-browsers (with no need for external programs) [9].

\section{LIBREFLIX}

Libreflix is an online platform for on-demand video created with the goal of providing free access to films of independent production. The platform allows users to browse and discover works of fiction, documentaries, and series to be watched on a variety of devices (browser, desktop, or mobile). Creators, directors and producers can use the platform for publishing and dissemination of their cultural pieces. The submission process for video content is collaborative, and starts with the creator filling a form on the website. Content management and curation is done collaboratively with the help of moderators with access to the administrative interface.

\subsection{Architecture}

Figure 1 shows the interactions among elements of the architecture of the Libreflix service. The website was developed in Node.js using the Model-View-Controller (MVC) design pattern. The users access the services through their devices and interact with several front-end technologies (bottom of the figure). Technologies such as Bootstrap and the template engine Nunjucks improve the user experience, providing visually appealing interfaces that are responsible to different screen sizes.

A mobile application is being developed to complement the webbased service. The implementation uses the Kotlin [8] language, which is a modern alternative for application development for Android (Figure 2).
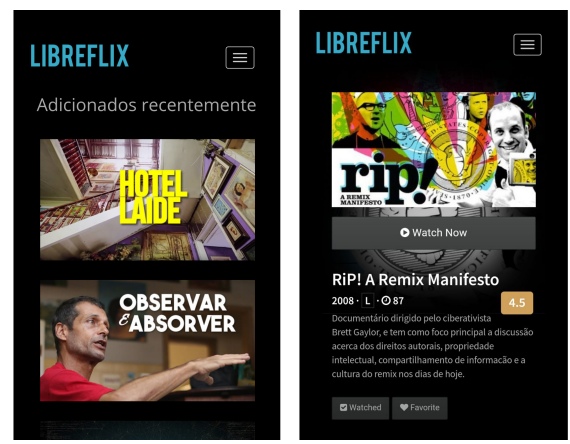

Figure 2: Screens of the mobile app

In the back-end, video processing is handled by a series of scripts using the powerful ffmpeg ${ }^{2}$ tool while P2P distribution is delegated to a set of customizations based on WebTorrent. Data storage and management is performed by a MongoDB instance which implements a NoSQL hierarchical data model. Elasticsearch [4], which offers a powerful and scallable search engine, was used to provide keyword-based queries for users to retrieve titles.

Following its commitment with free access, the platform uses the free Git versioning service LibreGit ${ }^{3}$. The Libreflix platform and its microservices are hosted on a virtual private server (VPS) running Debian GNU/Linux.

\subsection{Video Streaming}

The main goal of a video streaming platform is to provide a convenient watching experience for any title, at any time, and on any device. In Libreflix, the Watch interface houses the media player for content display. Initially, the standard media player was implemented to interface with the YouTube's Player API. This approach

\footnotetext{
${ }^{2}$ https://ffmpeg.org ${ }^{3}$ https://libregit.org
} 


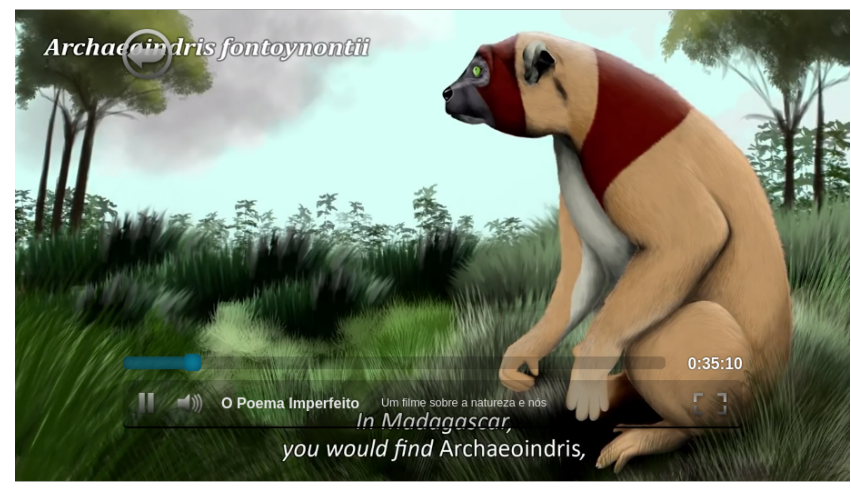

Figure 3: Documentary film playing within the platform

allows the use of YouTube's transmission resources and was important at the beginning when Libreflix did not have means to handle video delivery. Another advantage is the wide offer of titles that can be included in the catalog (since YouTube is a popular platform for creators)

Libreflix is currently on an effort to offer other streaming options. A new model recently incorporated in the platform is a $\mathrm{P} 2 \mathrm{P}$ protocol based on WebTorrent. Since both WebTorrent and Libreflix employ the Javascript programming language, it was easy to integrate the projects.

In the WebTorrent, data (video) chunks are transmitted to the client. The library renders the content directly in the browser's DOM (Document Object Model) for the video page. One issue with this approach (also common to other P2P implementations) is that the content must be shared by other users at the time of playback, requiring these users to be seeding the content or watching the same film.

A later development in the BitTorrent technology, called WebSeed, allows new peers to share the content through standard HTTP/FTP protocols [5]. These peers can then distribute data when there are not enough regular peers sharing the content. This approach has been integrated in WebTorrent as well, and was implemented in the Libreflix project.

This allows Libreflix to offer a hybrid data transmission strategy that employs seed servers whenever the number of regular peers is insufficient for high data throughput. Seed servers can be common web servers that support the network as peers and can be raised by the community related to the project, by volunteers, or other organizations interested in strength the project.

\section{USER AND COMMUNITY FEATURES}

This section discusses the main features offered in Libreflix with implementation details and the lessons learned in their development and deployment.

\subsection{Catalog browsing}

Libreflix's home page offers an exploratory interface for users to browse the available films (Figure 4). The titles are organized by several criteria such as publication time, popularity, format, duration,

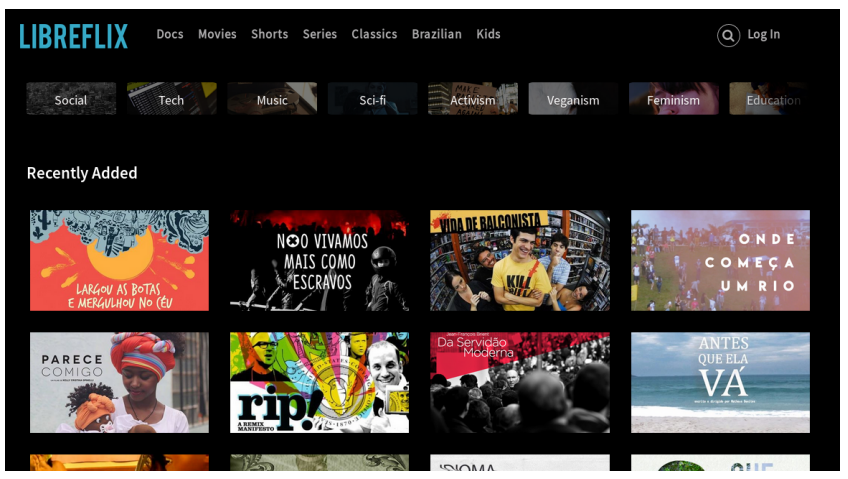

Figure 4: Platform home screen

category or tags. The menu offers links to the most accessed categories such as documentaries, fiction, short films, series, classics, and family/kids content.

By hovering the mouse over a film's cover, the user is presented with more information about the title, such as name, director, parental rating, and duration. Clicking on the cover opens a modal window with more information on the synopsis and attributed tags for the title.

Categorization of items is challenging for any digital library. In Libreflix the categorization is centered on user convenience, in a two-level hierarchy. The top level contains the main film formats: fiction, documentaries and series. While this division does not correlate with title type or duration, it is is more likely to match user's general intent in a watching session. From the top-level categories the users access sub-categories based on the format chosen. Examples of sub-categories are teen movies (fiction), educational (documentaries), and adventure (series).

Other options for navigation were explored trough the creation of specific URL slugs. The first one allows direct access to all titles available with a certain duration length limit. This feature can be used to show films according to the available time to watch of an user. The second slug refers to a page that will show all works from a specified country, allowing the direct access to other countries audiovisual culture.

Libreflix offers a search-based interface where users can use keywords to retrieve related titles. Submitted queries can include the name of the production, direction, category, format or key words at the sinopse.

Libreflix also offers a title recommendation engine, which suggests titles based on users' previous interactions. The recommendation engine has been implemented following studies on privacypreserving recommendation models [11]. Currently the recommendations use a collaborative filtering approach that will improve as users rate and interact with more titles.

\subsection{Title interface and community engagement}

When a user chooses a title she is redirected to a page containing detailed information about the work (Figure 5). This page also concentrates several community interaction features to foster user interaction, engagement, debate and networking. The main features are: 


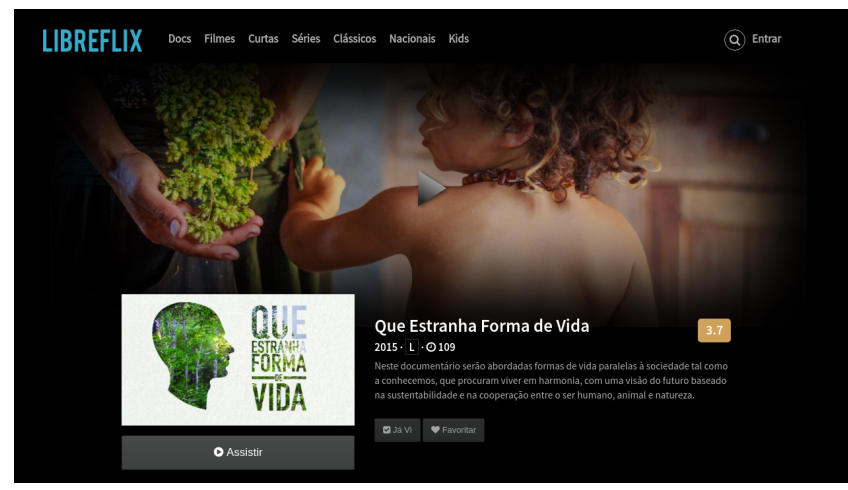

Figure 5: Page containing information about the work and enabling user interaction

- Comments field: for users to express their opinion on the title, write a synopsis, etc.

- Ratings: for users to assign a personal rating, using the 1 to 5 stars scheme often adopted in the cinema world. This data is used to calculate a global rating for each title by averaging all of its ratings.

- Favorite and Watched tags: users can use these two tags for personal organization. This data is also used by the backend in the recommender system. When a user rates a film, the film is automatically tagged as watched.

- External references: this feature allows external sources that cited the title to be displayed alongside the details for the work. External references can provide access to related synopses, news articles, reference sources, etc. The feature strengthen network connections, providing users with direct access to other perspectives and to the entire blogsphere.

- External links: this feature is intended to offer further institutional information about the title, such as official website, social network profiles, Wikipedia page, IMDB, etc. The links are an invitation for users to get to know and connect with the production.

\subsection{Administrative interface}

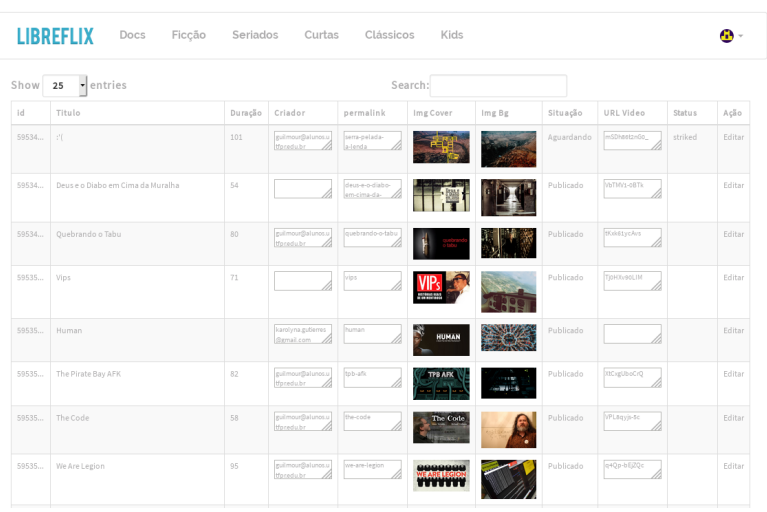

Figure 6: Administrative area for curation
Libreflix uses a collaborative approach to catalog curation. The administrative interface (Figure 6) handles most of the collaborative workflow, encompassing title submission, approval, metadata reviews, and publication. The main roles in the administrative interface are:

(1) Users submitting titles: usually the creator of the work (or someone that know the permissive licensing of it). The user fills a form with basic information about the work. In the submission page the user can follow the status of the review process and edit the metadata before the title is approved or rejected.

(2) Moderators: Moderator users have access to an interface with all titles submitted to the platform (Figure 6). The titles are organized in a spreadsheet containing key information such as work title, cover image, duration, posted by, etc. The moderator can contact users to obtain further information about their submissions. All moderators have permission to accept or reject submissions following Libreflix's guidelines. Any status change for a title is reported in an automatic email sent to the respective user.

\subsection{Internationalization}

A key factor for cultural interchange is the internationalization of a software or platform. According to [3], the internationalization process (i18n) is associated with the implementation of software resources to support translation and localization (110n). Translation refers to the literal conversion of text from one language to another. Localization refers to the adaptation of content to meet regional expectations when the content is displayed.

Libreflix supports internationalization in the main components of the user interface. The Node.js library i18n is used to handle automatic translation while browser cookies store user's language preferences.

To allow users with no technical background to help in the translation process, Libreflix uses the Weblate ${ }^{4}$ project, which allows translations to be created, discussed, compared and approved in a web-based interface. Weblate supports exporting the translations to JSON which, combined with Libreflix Git-based and open source code development, allows for streamlined publication of the translated sections.

\section{FINANCE, LICENSING AND PERSPECTIVES}

This section discusses important aspects of Libreflix's financing strategy, licensing model and contextualizes the current and future social impact of the platform.

\subsection{Financing model}

Libreflix advocates a free access model to all content served. To support the service Libreflix accepts voluntary donations from users using the platform Acredito.me $\mathrm{e}^{5}$ which allows the publication of personalized crowdfunding campaigns without retaining fees other than payment gateways.

It is also important to implement mechanisms to help in generating funding for the creators. Libreflix allows, for each registered title, a monetary retribution option to be displayed in the details

\footnotetext{
${ }^{4}$ https://weblate.org ${ }^{5}$ https://acredito.me
} 
page. When a user wants to make a donation for the production of a title, a pop-up window is shown where she can set the value of the contribution (Figure 7). The user is then redirected to services like PayPal and Pagli to continue the transaction.

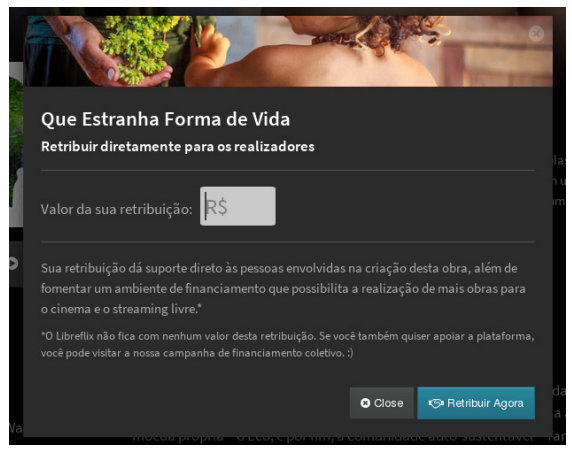

Figure 7: Pop-up window to contribute with the production

\subsection{Licensing model}

The license in which Libreflix is distributed is the GNU Affero General Public License (AGPLv3) which makes explicit the need for access to source code even when there is no direct access to the application's executable binary (including web applications). Mobile and desktop applications also follow licenses in the free software philosophy.

The choice of license permeates all aspects of Libreflix, fostering Free Streaming and more independent content distribution. This model provides the possibility of a community process of planning, development, and learning. It also allows users to create their instances and adapt them their needs, ensuring that their improvements will be public for others. The source code for the platform can be accessed on https://libregit.org/libreflix.

\subsection{Perspectives}

The feedback on the Libreflix platform has been very positive. Even though registration is not mandatory, the number of registered users has surpassed 30,000. The most recent statistics (first half of 2019) shows average visits counts of around 85,000 per month.

The catalog has over 310 available works, where $75 \%$ are nonfiction works and $62 \%$ correspond to works produced in Brazil. $40 \%$ are feature films, $57 \%$ shorts and $3 \%$ are series. The moderators are constantly working to correctly process, approve, and manage the incoming submissions. The number of pending works is approximately 250 .

The project's goal now is to reach more people, fostering free streaming and the dissemination of digital culture. Next steps include the development of a Smart TV application and enhancements on the mobile app.

Another interesting project is the creation of an offline version, which will allow the platform to run on community networks where internet access are unavailable or limited. Alongside that, we hope to gather more volunteers to help on moderation, translation of the software and subtitle creation.

\section{CONCLUSION}

This paper presented Libreflix, and on-demand, free and collaborative video streaming platform. Libreflix has evolved to become a service that integrates several tools and strategies to foster the dissemination of the digital multimedia culture. The platform is becoming a reference point for independent creators as well as consumers interested in alternative content.

By using decentralized technologies, such as P2P data sharing, the platform offers an alternative distribution paradigm giving users an appealing option beyond traditional providers and illegal (pirated) content.

Another important concern in the development of Libreflix is to integrate means for direct and voluntary support to creators. In a streamlined fashion, users can choose to contribute with small monetary donations directly to content producers.

The main instance of the Libreflix platform can be accessed in the address: https://libreflix.org. Demo and tutorial videos are available on: https://vdn.libreflix.org/demo/demo.html.

\section{ACKNOWLEDGMENTS}

This work has been supported with funding from the Laboratory of Free Technologies (LabLivre) through the Research Development Foundation (Fundep) - Grant/Edital 01/2018 LabLivre UFABC. We would also like to thank the users that voluntarily helped in Libreflix's crowdfunding campaign.

\section{REFERENCES}

[1] Feross Aboukhadijeh. 2014. WebTorrent.

[2] VNI Cisco. 2018. Cisco visual networking index: Forecast and trends, 2017-2022. White Paper 1 (2018).

[3] Joao Dias e Vitor Gonçalves. 2017. Localização de Software e Páginas Web. adolesCiência 4, 1 (2017), 85-90. https://www.adolesciencia.ipb.pt/index.php/ adolesciencia/article/view/197

[4] Elastic. 2015. Hardware: Elasticsearch: The Definitive Guide. https://www. elastic.co/guide/en/elasticsearch/guide/current/hardware.html

[5] John Hoffman and DeHackEd. 2008. HTTP Seeding - BitTorrent Enhancement Proposal No 17. http://www.bittorrent.org/beps/bep_0017.html

[6] James F. Kurose and Keith W. Ross. 2006. Redes de computadores e a Internet: uma nova abordagem. $3^{\text {a }}$ edição.

[7] João Carlos Massarolo and Dario Mesquita. 2017. Video on demand: a new television platform.

[8] Kassiano Resende. 2018. Kotlin com Android: Crie aplicativos de maneira fácil e divertida. Casa do Código.

[9] Roberto Oliveira Rocha. 2014. WebRTC - Evolução na Web.

[10] Guilmour Rossi. 2019. Free Streaming: Thinking on a Digital Distribution of the Commons in the Streaming Era. In Anais do I Congresso Internacional sobre o Comum e os Commons. to appear.

[11] Guilmour Rossi, Luiz Gomes-Jr, Marcelo Rosa, and Keiko Fonseca. 2018. Privacypreserving recommendations for Online Social Networks using Trusted Execution Environments. In Anais do XVIII Simpósio Brasileiro em Segurança da Informação e de Sistemas Computacionais. SBC, Porto Alegre, RS, Brasil, 41-48. https://sol. sbc.org.br/index.php/sbseg/article/view/4268

[12] Richard Stallman. 2002. Free software, free society: Selected essays of Richard M. Stallman. GNU Press. https://www.gnu.org/philosophy/fsfs/rms-essays.pdf 